\title{
DYNAMICAL STABILIZERS AND COUPLED SYSTEMS
}

\author{
FARID AMMAR KHODJA, ASSIA BENABDALLAH, AND DJAMEL TENIOU
}

Abstract. Given a linear (unbounded) operator $A$ in a Hilbert space, we describe classes of operators $B$ and $C$ which allow strong or exponential stability of the system $u^{\prime}=A u+B v / / / v i=-B * u+C v$.

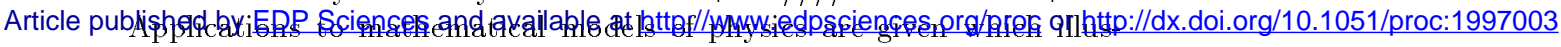
trate our results.

Key words: Stabilization, Thermoelasticity

Mathematics subject classification: 73B30, 93D15, 93D20.

\section{INTRODUCTION}

Let's consider the equation

$$
u^{\prime}(t)=A u(t)+B v(t)
$$

where $(A, D(A))$ is a linear operator on a Hilbert space $X$ which is an infinitesimal generator of a $C_{0}$-semigroup and $B$ is a closed linear operator from a Hilbert space $Y$ to $X$. Our problem is to find, among linear (unbounded) operators $(C, D(C))$ which are generators of $C_{0}$-semigroups on $Y$, those which insure the exponential or strong decay of the energy of the system

$$
\left\{\begin{array}{c}
u^{\prime}(t)=A u(t)+B v(t) \\
v^{\prime}(t)=-B^{*} u^{\prime}(t)+C v(t) \\
u(0)=u_{0}, \quad v(0)=v_{0}
\end{array}\right.
$$

as $t$ goes to infinity. A pair $(B, C)$ which satisfies this property will be called a dynamical stabilizer of $A$. In the finite dimensional case, this problem has been intensively studied and we refer the interested reader to [8].

This paper is organized as follows. In the second section, we study the effect of the pair $(B, C)$ whenever the semigroup $S_{A}(t)$ generated by $A$ is initially uniformly stable: sufficient conditions on $(B, C)$ are given which allow conservation of the uniform stability property. The third section is devoted to the case where $S_{A}(t)$ is not uniformly stable. In the first subsection, the strong stability is studied while the second subsection deals with uniform stabilization. Each of our theoretical results is applied to mathematical models of physics (linear thermoelasticity and thermoplate systems or closely related systems). The proofs of the results are omitted but we indicate references where they may be found.

\section{Coupling Two Uniformly Stable EQUATIONS}

In this section, we study the effect of the coupling operator $B$ on two uniformly stable dynamics. More precisely, we set the following assumption: 


\section{Assumption (E.S)}

1)- $(A, D(A))$ is a generator of an exponentially stable semigroup of contractions $\left(S_{A}(t)\right)_{t}$ on the Hilbert space $X$ : there exists $\omega_{A}>0$ and $M_{A}>0$ such that

$$
\left\|S_{A}(t)\right\|_{X} \leq M_{A} e^{-t \omega_{A}} \quad \forall t \geq 0
$$

2)- $(C, D(C))$ is self-adjoint on the Hilbert space $Y$ and generator of an exponentially stable semigroup $\left(S_{C}(t)\right)_{t}$.

$3)-(B, D(B))$ is an operator from $Y$ to $X$ such that $D(A) \subset D\left(B^{*}\right)$ and it is $(-C)^{\frac{1}{2}}$ - bounded, i.e: $D\left((-C)^{\frac{1}{2}}\right) \subset D(B)$ and

$$
B(-C)^{-\frac{1}{2}} \in L(Y, X)
$$

4)- The operator

$$
L=\left(\begin{array}{cc}
A & B \\
-B^{*} & C
\end{array}\right)
$$

with $D(L)=D(A) \times D(C)$ is closed and there exists $\lambda_{0}>0$ such that $\left(\lambda_{0} I-L\right)$ is into.

Proposition 1. Under assumption (E.S), the operator $L$ is an infinitesimal generator of a $C_{0}$-semigroup $S_{L}(t)$ of contractions.

TheOREM 2. The semigroup $S_{L}(t)$ generated by $(L, D(L))$ is exponentially stable: there exist $\omega>0$ and $M>0$ such that

$$
\left\|S_{L}(t)\right\|_{X \times Y} \leq M e^{-\omega t} \quad \forall t \geq 0
$$

For the proof, see [5]. An example where $C$ is nonselfadjoint is given in $[6]$

\section{Example 1.}

Let's consider the system

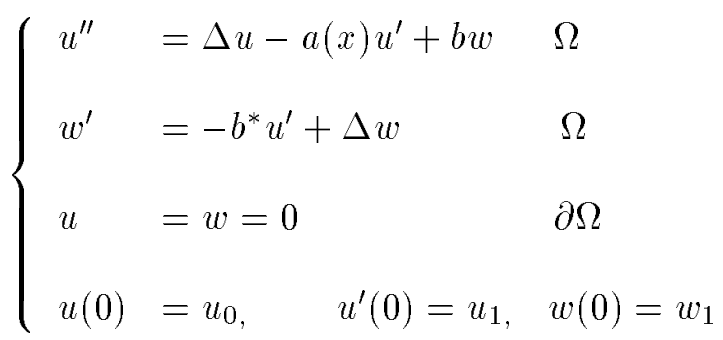

where $\Omega$ is an open bounded subset of $R^{n}$ with a smooth boundary, $a \in$ $L^{\infty}\left(\Omega, R^{+}\right)$and $(b, D(b))$ is a linear operator on $L^{2}(\Omega)$ which will be precised later. System $(2)$ can be written formally:

$$
\left\{\begin{array}{c}
Y^{\prime}=L Y \\
Y(0)=Y_{0}=\left[\begin{array}{c}
u_{0} \\
u_{1} \\
w_{0}
\end{array}\right]
\end{array}\right.
$$

with:

$$
L=\left(\begin{array}{cc}
\left(\begin{array}{cc}
0 & I \\
\Delta & -a
\end{array}\right) & \left(\begin{array}{l}
0 \\
b
\end{array}\right) \\
\left(\begin{array}{cc}
0 & -b^{*}
\end{array}\right) & \Delta
\end{array}\right)=\left(\begin{array}{cc}
A & B \\
-B^{*} & C
\end{array}\right)
$$


defined on $H=H_{0}^{1}(\Omega) \times L^{2}(\Omega) \times L^{2}(\Omega)$ (the energy space equipped with the product norm denoted by $\|\cdot\|)$ and

$$
\begin{aligned}
D(L) & =D(A) \times D(C) \\
& =\left(H^{2}(\Omega) \cap H_{0}^{1}(\Omega)\right) \times H_{0}^{1}(\Omega) \times\left(H^{2}(\Omega) \cap H_{0}^{1}(\Omega)\right)
\end{aligned}
$$

Then, as a direct consequence of the previous result (Theorem 2):

Theorem 3. If :

$$
a \geq a_{0}>0 \text { a.e on } \Omega_{0} \subset \Omega
$$

where $\Omega_{0}$ is open and satisfies the geometrical control property and $b$ is $(-\Delta)^{\frac{1}{2}}$-bounded, then $\left(S_{L}(t)\right)_{t \geq 0}$ is exponentially stable: there exist $\omega>0$ and $M>0$ such that :

$$
\left\|S_{L}(t)\right\| \leq M e^{-\omega t} \quad \text { for all } t \geq 0
$$

Proof: From the results of [7], $A$ generates an exponentially stable semigroup. The other assumptions in (E.S) may be easily verified.

Let's now recall that the type of the semigroup $S_{L}(t)$ is given by

$$
\omega(L):=\lim _{t \rightarrow+\infty} \frac{\ln \left\|S_{L}(t)\right\|}{t}
$$

Proposition 4. If $a>0$ and $b$ are constants, then the eigenvalues $\left(\lambda_{3 k+j}\right)$ of $L$ satisfy the following properties :

(i)

$$
\begin{array}{rlrl}
\lambda_{3 k} & >-\mu_{k} & & k \geq 1 \\
\lim _{k \rightarrow+\infty} \operatorname{Re}\left(\lambda_{3 k+j}\right) & =-\frac{a}{2} & j=1,2
\end{array}
$$

(ii) For all $b \in R^{*}$, there exists $\left.a_{0}=a_{0}(b) \in\right] 0, \sqrt{2 \mu_{1}}[$ such that

$$
\begin{aligned}
\omega(L) & =\omega(A) \quad \text { if } \quad a<a_{0} \\
& \geq \omega(A) \quad \text { if } \quad a>a_{0}
\end{aligned}
$$

where the $\mu_{k}$ are the eigenvalues of $(-\Delta)$ (with Dirichlet boundary condition) and

$$
\omega(A)=\left\{\begin{array}{l}
-\frac{a}{2} \quad \text { if } a \leq \sqrt{2 \mu_{1}} \\
-\frac{a}{2}+\frac{\sqrt{a^{2}-4 \mu_{1}}}{2} \quad \text { if } \quad a>\sqrt{2 \mu_{1}}
\end{array}\right.
$$

REMARK 5. This last proposition proves that one cannot expect uniform stability whenever $a=0$ since, in this case, (5) and the Hille-Yosida theorem imply that $\omega(L)=0$ (see [1] and [2]). Let's also point out that, in the situation described by the proposition ( $a$ and $b$ constants), the type of $S_{L}(t)$ is larger than the one of $S_{A}(t)$. It means that, when $b$ is bounded, the type of $S_{A}(t)$ is not in general improved once the corresponding equation is coupled to give system (1). 


\section{Dynamical stabilization}

This section is devoted to the situation where $S_{A}(t)$ is not initially stable. We will give sufficient conditions on $B, C$ to stabilize (1). Of course, we will study separately strong and uniform stability. It will appear, in the second subsection, that uniform stabilization for system (1) requires unboundedness for the operator $B$.

\subsection{Strong stabilization}

Definition 6. A $C_{0}$-semigroup $(S(t))_{t}$ in a Banach space $E$ is strongly stable if

$$
\lim _{t \rightarrow+\infty} S(t) x=0 \quad \forall x \in E
$$

In this section, assumption (E.S) is replaced by:

\section{Assumption (A.S)}

1)- $(A, D(A))$ has a compact resolvent and is a generator of contraction semigroup $\left(S_{A}(t)\right)_{t}$ on the Hilbert space $X$, .

2)- $(C, D(C))$ has a compact resolvent, is self-adjoint on the Hilbert space $Y$ and generator of an exponentially stable $C_{0}-\operatorname{semigroup~}\left(S_{C}(t)\right)_{t}$.

3)-i) $D(C) \subset D(B)$ and there exist two constants $\alpha_{C}$ and $\delta_{C}$ with

$$
0 \leq \alpha_{C}<1 \quad, \quad \delta_{C} \geq 0
$$

such that:

$$
\|B v\|_{X} \leq \alpha_{C}\|C v\|_{Y}+\delta_{C}\|v\|_{Y}
$$

-ii)

$$
D(A) \subset D\left(B^{*}\right)
$$

and there exist two constants $\alpha_{A}$ and $\delta_{A}$ with:

$$
0 \leq \alpha_{A}<1, \quad \delta_{A} \geq 0
$$

such that:

$$
\left\|B^{*} u\right\|_{Y} \leq \alpha_{A}\|A u\|_{X}+\delta_{A}\|u\|_{X}
$$

REMARK 7. Assumption (A.S $\mathrm{S}_{3}$ ) is natural because the operator $L$ can be written:

$$
L=D+E
$$

with

$$
D=\left(\begin{array}{cc}
A & 0 \\
0 & C
\end{array}\right), \quad E=\left(\begin{array}{cc}
0 & B \\
-B^{*} & 0
\end{array}\right)
$$

Clearly $D$ is a generator of contraction semigroup and since $E$ is dissipative, $L$ is also a generator of contraction semigroup

THEOREM 8. Under assumption (A-S), if the unique solution of the system

$$
\begin{aligned}
& u^{\prime} \quad=A u \quad t \geq 0 \\
& B^{*} u=0 \quad t \geq 0
\end{aligned}
$$

is $u \equiv 0$, then $S_{L}(t)$ is asymptotically stable.

(see [5] for the proof which is essentially Lassalle's principle). 
REMARK 9. In fact, one can relax the hypothesis on $C$. More precisely, one may drop the selfadjointness of $C$ and suppose

$$
\operatorname{Re}(C v, v)=0 \Rightarrow v=0
$$

(see $[6])$.

\section{Example 2.}

1/ First, we consider system (2). One has:

Proposition 10. If $b$ is $(-\Delta)^{\frac{1}{2}}$-bounded and $a \equiv 0$ a.e. in $\Omega$, then for all $Y_{0} \in X:$

$$
\left\|S_{L}(t) Y_{0}\right\| \rightarrow 0 \text { as } t \rightarrow+\infty
$$

provided that the unique solution of

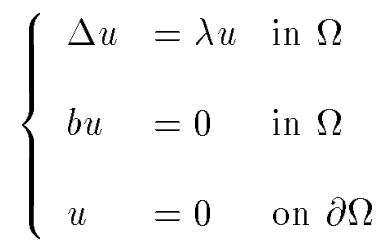

is $u \equiv 0$ for all $\lambda \in R$.

This result is in particular true if $b$ is a bounded operator in $L^{2}(\Omega)$. In this case, one cannot expect the exponential stability for if $a \equiv 0$ and $b$ is a constant, this is not true (see Remark 1).

$2 /$ The second example deals with the linear thermoelasticity system. Let $\Omega$ an open bounded set in $R^{n}$ with smooth boundary $\Gamma$,

$$
\begin{cases}u^{\prime \prime}=\Delta u+\alpha \nabla w & R^{+} \times \Omega \\ w^{\prime}=\Delta w+\alpha \nabla \cdot u^{\prime} & R^{+} \times \Omega \\ u=0 & R^{+} \times \Gamma \\ w=0 & R^{+} \times \Gamma \\ u(0)=u_{0}, u^{\prime}(0)=u_{1}, w(0)=w_{0}, & \Omega\end{cases}
$$

where $u=\left[\left(u_{1}, \ldots, u_{n}\right)\right]$ (resp. $\left.w\right)$ is the displacement (resp. the temperature) of the system, $\alpha>0$ is the coupling parameter, (see [11]).

The energy space will be the Hilbert space:

$$
\left.H=H_{0}^{1}(\Omega)\right)^{n} \times\left(L^{2}(\Omega)\right)^{n} \times L^{2}(\Omega)
$$

with the scalar product:

$$
<\left[\begin{array}{c}
u \\
v \\
w
\end{array}\right],\left[\begin{array}{l}
f \\
g \\
h
\end{array}\right]>=\int_{\Omega}(\nabla u . \nabla f+v g+w h) d x
$$

and we denote by $\|$.$\| the induced norm on H$. |.| will denote the $L^{2}$-norm.

Let:

$$
L=\left(\begin{array}{cc}
\left(\begin{array}{cc}
0 & I \\
\Delta & 0
\end{array}\right) & \left(\begin{array}{c}
0 \\
\alpha \nabla
\end{array}\right) \\
\left(\begin{array}{cc}
0 & \alpha \nabla .
\end{array}\right) & \Delta
\end{array}\right)=\left(\begin{array}{cc}
A & B \\
-B^{*} & C
\end{array}\right)
$$




$$
\begin{aligned}
D(L) & =D(A) \times D(C) \\
& =\left(H^{2}(\Omega) \cap H_{0}^{1}(\Omega)\right)^{n} \times H_{0}^{1}(\Omega)^{n} \times\left(H^{2}(\Omega) \cap H_{0}^{1}(\Omega)\right)
\end{aligned}
$$

One has

PROPOSITION 11. The thermoelasticity system is strongly stable if the system

$$
\left\{\begin{array}{lll}
u^{\prime \prime} & =\Delta u & \Omega \\
u & =0 & \partial \Omega \\
\nabla \cdot u^{\prime} & =0 & \Omega
\end{array}\right.
$$

has $u \equiv 0$ as a unique solution.

This result was already known ([9]) and we give it for illustration.

\subsection{UNIFORM STABILIZATION}

As we are interested by equations closely related to the wave equation, we restrict ourselves to the stabilization of second order equations. More precisely, let $X$ and $Y$ be two Hilbert spaces and $A, B$ and $C$ are unbounded operators, with dense domains, acting on $X$, from $Y$ to $X$ and on $Y$ respectively. We consider the system:

$$
\left\{\begin{array}{lll}
u^{\prime \prime}(t)+A u(t)=B w(t) & t \in R^{+} \\
w^{\prime}(t)+C w(t)=-B^{*} u^{\prime}(t) & t \in R^{+} \\
u(0)=u_{0} ; & u^{\prime}(0)=v_{0} ; & w(0)=w_{0}
\end{array}\right.
$$

where $B^{*}$ is the adjoint operator of $B$.

Throughout, we will set two kind of hypothesis. The first one will insure the semigroup property for the system (assumption (H1)). The second will insure the uniform (exponential) stability for the semigroup solution (assumption (H2)).

(H.1) (i) $A$ (resp. $C$ ) is a self-adjoint operator on a Hilbert space $X$ (resp. $Y$ ), strictly positive, with dense domain $D(A)$ (resp. $D(C)$ ) and compact resolvents;

(ii) $B$ (resp. $B^{*}$ ) is an operator from $Y$ to $X$ (resp. $X$ to $Y$ ) such that: $D(C) \subset D(B)$ (resp. $D(A) \subset D\left(B^{*}\right)$ ) and is $C$-bounded.

In the sequel, $H=D\left(A^{\frac{1}{2}}\right) \times X \times Y$ will be equipped with the inner product:

$$
\left(\left[\begin{array}{c}
u \\
v \\
w
\end{array}\right],\left[\begin{array}{l}
f \\
g \\
h
\end{array}\right]\right)=\left(A^{\frac{1}{2}} u, A^{\frac{1}{2}} f\right)_{X}+(v, g)_{X}+(w, h)_{Y}
$$

The preceding system can be equivalently written:

$$
\left\{\begin{array}{l}
Y^{\prime}(t)=L Y(t) \quad t \in R^{+} \\
Y(0)=Y_{0}
\end{array}\right.
$$




$$
\begin{aligned}
L & =\left(\begin{array}{ccc}
0 & I & 0 \\
-A & 0 & B \\
0 & -B^{*} & -C
\end{array}\right) \\
D(L) & =D(A) \times\left(D\left(A^{\frac{1}{2}}\right) \cap D\left(B^{*}\right)\right) \times D(C)
\end{aligned}
$$

Since $L$ is dissipative with dense domain in $H, L$ is closable. So, the closure of $L$ (and its closure will be denoted by the same symbol) is a generator of a $C_{0}$-semigroup of contractions.

(H.2) (i)

$$
\left\|A^{-\frac{1}{2}} B w\right\|_{X \leq a}\left\|C^{\frac{1}{2}} w\right\|_{Y} \quad \forall w \in D(C) ;
$$

(ii) $B^{*}$ is invertible $\left(\left(B^{*}\right)^{-1} \in L(X, Y)\right)$ and

$$
\left\|\left(B^{*}\right)^{-1} C w\right\|_{X} \leq \quad b\left\|C^{\frac{1}{2}} w\right\|_{Y} \quad \forall w \in D(C) ;
$$

$a$ and $b$ being positive real constants.

(iii) There exist three positive constants $c_{1}, c_{2}, c_{3}$ such that for all $Y \in$ $D(L)$

$$
\left|\left(A u,\left(B^{*}\right)^{-1} w\right)_{X}\right| \leq \frac{c_{1}}{2 \alpha}\left\|C^{\frac{1}{2}} w\right\|_{Y}^{2}+\frac{\alpha}{2}\left(c_{2}\|Y\|^{2}+(G(Y), L Y)\right)
$$

for all $\alpha>0$, where $G$ is a function from $H$ to $L(H)$ which satisfies

$$
\|G(Y)\| \leq c_{3}\|Y\| \quad \forall Y \in H
$$

The main result is then:

THEOREM 12. Under assumption (H), $L$ is the generator of a uniformly stable $C_{0}$-semigroup $\left(S_{L}(t)\right)$ : there exist $\omega>0$ and $M \geq 1$ such that:

$$
\left\|S_{L}(t)\right\|_{L(H)} \leq M e^{-\omega t} \quad \forall t \in R^{+}
$$

A particular system of this kind is obtained if one sets $X=Y, A=C$ and $B=A^{\alpha}$ where $\alpha \in[0,1]$. Denoting by

$$
\begin{gathered}
L_{\alpha}=\left(\begin{array}{ccc}
0 & I & 0 \\
-A & 0 & A^{\alpha} \\
0 & -A^{\alpha} & -A
\end{array}\right) \\
D\left(L_{\alpha}\right)=D(A) \times\left(D\left(A^{\alpha}\right) \cap D\left(A^{\frac{1}{2}}\right)\right) \times D(A)
\end{gathered}
$$

we show the following:

THEOREM 13. (i) The strongly continuous semigroup of contractions $\left(S_{\alpha}(t)\right)$ generated by $L_{\alpha}$ is uniformly stable if and only if $\alpha \in\left[\frac{1}{2}, 1\right]$. In this case, the decay rate (or the type) is $\omega=-\sup \operatorname{Re} \sigma\left(L_{\alpha}\right), \sigma\left(L_{\alpha}\right)$ being the spectrum of $L_{\alpha}$.

(ii) If $\alpha \in\left[0, \frac{1}{2}\left[, S_{\alpha}(t)\right.\right.$ is strongly stable.

(iii) The semigroup $S_{\alpha}(t)$ is analytic if and only if $\frac{3}{4} \leq \alpha \leq 1$.

(iv) The semigroup is compact if and only if $\frac{1}{2}<\alpha \leq 1$.

This result shows the "order" of unboundedness of $B$ with respect to $A$ and $C$ which insures uniform stability. 
EXAMPLE 14. We consider two models of thermoelastic plates which differ by the boundary conditions. Let $\Omega$ be an open bounded set in $R^{n}$ with smooth boundary $\Gamma$,

$$
\begin{cases}u^{\prime \prime}=-\Delta^{2} u-\Delta w & R^{+} \times \Omega \\ w^{\prime}=\Delta w+\Delta u^{\prime} & R^{+} \times \Omega \\ u=\Delta u=0 & R^{+} \times \Gamma \\ w=0 & R^{+} \times \Gamma \\ u(0)=u_{0}, u^{\prime}(0)=u_{1}, w(0)=w_{0}, & \Omega\end{cases}
$$

The energy space will be the Hilbert space:

$$
H=\left(H^{2}(\Omega) \cap H_{0}^{1}(\Omega)\right) \times L^{2}(\Omega) \times L^{2}(\Omega)
$$

with the scalar product:

$$
<\left[\begin{array}{c}
u \\
v \\
w
\end{array}\right],\left[\begin{array}{l}
f \\
g \\
h
\end{array}\right]>=\int_{\Omega}(\Delta u . \Delta f+v g+w h) d x
$$

and we denote by $\| .||$ the induced norm on $H$. |.| will denote the $L^{2}$-norm.

Let:

$$
\left.\begin{array}{c}
\left.L=\left(\begin{array}{cc}
0 & I \\
-\Delta^{2} & 0
\end{array}\right)\left(\begin{array}{c}
0 \\
-\Delta
\end{array}\right)\right) \\
\left(\begin{array}{cc}
0 & \Delta
\end{array}\right)
\end{array}\right)
$$

This model has already been studied in [14] where uniform stability is proved. This system satisfies Assumption $(\mathrm{H})$ with $A=\Delta^{2}$ and

$$
D(A)=\left\{u \in H^{2}(\Omega) \cap H_{0}^{1}(\Omega) ; \Delta u \in H^{2}(\Omega) \cap H_{0}^{1}(\Omega)\right\}
$$

$C=B=A^{\frac{1}{2}}=-\Delta$ with $D(C)=D(B)=H^{2}(\Omega) \cap H_{0}^{1}(\Omega)$ and $F=0$. So

Proposition 15. The thermoelastic plates system (11) is uniformly stable. Moreover, the semigroup associated to $L$ is compact and the decay rate of the energy is $\sup \operatorname{Re} \sigma(L)$.

The second model is the following

$$
\begin{cases}u^{\prime \prime}=-\Delta^{2} u-\Delta w & R^{+} \times \Omega \\ w^{\prime}=\Delta w+\Delta u^{\prime} & R^{+} \times \Omega \\ u=\frac{\partial u}{\partial \nu}=w=0 & R^{+} \times \Gamma \\ u(0)=u_{0}, u^{\prime}(0)=u_{1}, w(0)=w_{0}, & \Omega\end{cases}
$$

The energy space will be the Hilbert space:

$$
H=H_{0}^{2}(\Omega) \times L^{2}(\Omega) \times L^{2}(\Omega)
$$


with the scalar product:

$$
<\left[\begin{array}{c}
u \\
v \\
w
\end{array}\right],\left[\begin{array}{l}
f \\
g \\
h
\end{array}\right]>=\int_{\Omega}(\Delta u . \Delta f+v g+w h) d x
$$

and we denote by $\|$.$\| the induced norm on H$. |.| will denote the $L^{2}$-norm.

$$
\text { Let: }
$$

$$
\begin{gathered}
L=\left(\begin{array}{cc}
\left(\begin{array}{cc}
0 & I \\
-\Delta^{2} & 0
\end{array}\right)\left(\begin{array}{c}
0 \\
-\Delta
\end{array}\right) \\
\left(\begin{array}{cc}
0 & \Delta
\end{array}\right) & \Delta
\end{array}\right) \\
D(L)=\left\{\left[\begin{array}{c}
u \\
v \\
w
\end{array}\right] \in H ; \Delta u \in H^{2}(\Omega) v \in H_{0}^{2}(\Omega) w \in\left(H^{2}(\Omega) \hat{E} \cap H_{0}^{1}(\Omega)\right)\right\}
\end{gathered}
$$

This model has also been studied in [10] where it is proved that the system is uniformly stable using an indirect method. We obtain here the same result but with an estimate of the decay rate. Let's denote by $A=\Delta^{2}$ and $D(A)=$ $H^{4}(\Omega) \cap H_{0}^{2}(\Omega), C=B=-\Delta$ with $D(C)=D(B)=H^{2}(\Omega) \cap H_{0}^{1}(\Omega)$.

Proposition 16. The thermoelastic plates system (12) is uniformly stable.

In these two last models, the method we use to prove uniform stability is based on the construction of Lyapunov functions (using the multipliers method). It allows us to give an estimate of the decay rate of the energy.

The reader can find all the proofs of the results of this subsection in [3] and [4]. For more precisions on the definitions of the different concepts of stability, see[12], [13].

\section{REFERENCES}

[1] Ammar Khodja F. et Benabdallah A., Stabilisation de l'équation des ondes par un contrôleur dynamique, C. R. Acad. Sci Paris, 321, I, p. 195-198, 1995.

[2] F. Ammar Khodja and A. Benabdallah, Stabilization of the wave equation by a dynamical controller, Prépub. de l'équipe de Mathématiques de Besançon, 95/22.

[3] Ammar Khodja F. et Benabdallah A., Conditions suffisantes de stabilisation dynamique uniforme d'une équation du second ordre, C.R.A.S., 323, (1996), 615-620

[4] Ammar Khodja F. et Benabdallah A., Sufficient conditions for uniform stabilization of second order equations by dynamical controllers, Prépub. de l'équipe de Mathématiques de Besançon, 96/19.

[5] Ammar Khodja F., Benabdallah A. and Teniou D., Coupled systems, Abstract and Applied Analysis, Vol. 1, 3 (1996) 327-340.

[6] Ammar Khodja F., Benabdallah A. and Teniou D., Sur la stabilisation d'un couplage ondes-advection,Prépub. de l'équipe de Mathématiques de Besançon, 1996.

[7] C. Bardos, G. Lebeau and J. Rauch, Contrôle et stabilisation dans les problèmes hyperboliques, Appendix II in $\mathrm{Vol} 1$ of [J-L]

[8] Curtain R. F. and Zwart H. J., An Introduction to Infinite-Dimensional Linear Systems Theory, Texts in Applied Mathematics, 21, Springer-Verlag, 1995.

[9] Dafermos C., On the existence and asymptotic stability of solutions to the equations of linear thermoelasticity, Arch. Rat. Mech. Anal., vol 29, 4, 1968, 241-271.

[10] Kim J.U., On the energy decay of a linear thermoelasticitc bar and plate, SIAM J.Math.Anal, 23, 4, (1992), p. 889-899.

[11] J.-L. Lions, Contrôlabilité exacte, perturbations et stabilisation de systèmes distribués, Vol 1 et 2, RMA 9, Masson 1988.

[12] A. Pazy, Semigroups of linear operators and applications to partial differential equations, Applied mathematical sciences (Springer-Verlag) 1983 
[13] A. Pritchard and J. Zabczyk, Stability and stabilizability of infinite dimensional systems, SIAM Rev., 23 (1981) 25-61.

[14] R.Racke, Existence and regularity of solutions to nonlinear thermoelastic systems, Proceedings of the Workshop on Qualitative aspects and applications of nonlinear evolution equations, Trieste May 3-14, p. 74-87 1993

Farid Ammar Khodja et Assia Benabdallah : Université de Franche Comté Laboratoire de Calcul Scientifique et UMR 6623, 16 Route de Gray, 25030 Besançon, France ; Duamel Teniou : Institut de Mathématiques, Université Houari Boumediène, B.P. 3216111 El Alia, AlgÉrie 